\title{
Stable hypersurfaces in the complex projective space
}

\author{
Erika Battaglia $^{1} \cdot$ Roberto Monti $^{1}$ (D) Alberto Righini ${ }^{1}$
}

Received: 18 December 2018 / Accepted: 3 June 2019 / Published online: 10 June 2019

(c) Fondazione Annali di Matematica Pura ed Applicata and Springer-Verlag GmbH Germany, part of Springer Nature 2019

\begin{abstract}
We characterize the sphere with radius $\tan ^{2} r=2 n+1$ in the complex projective space $\mathbf{C} P^{n}$ as the unique stable hypersurface subject to certain bounds on the curvatures.
\end{abstract}

Keywords Stable hypersurfaces · Complex projective space $\cdot$ Constant mean curvature hypersurfaces

Mathematics Subject Classification 49Q10 · 53C42

\section{Introduction}

Barbosa et al. [2] proved among other results that the geodesic sphere with radius $r \in(0, \pi / 2)$ in the complex projective space $\mathbf{C} P^{n}, n \geq 2$, is stable for the area functional with fixed enclosed volume if and only if $\tan ^{2} r \leq 2 n+1$. They also computed the stability intervals for the radius of a geodesic tube around $\mathbf{C} P^{k} \subset \mathbf{C} P^{n}$, with $1 \leq k<n$.

The classification of complete oriented stable hypersurfaces in $\mathbf{C} P^{n}$ could be an important step toward the classification of isoperimetric sets in $\mathbf{C} P^{n}$. Indeed, the boundary of an isoperimetric set, if smooth, is a hypersurface with constant mean curvature that is stable for variations fixing the volume. Since [2], there was apparently no progress on the problem of the classification of stable hypersurfaces in $\mathbf{C} P^{n}$.

In this paper, we characterize the geodesic sphere with radius $\tan ^{2} r=2 n+1$ as the unique stable connected and complete hypersurface subject to a bound either on the characteristic curvature or on the restriction of the second fundamental form to the complex tangent space. See Definition 6.1 for the precise definition of stability.

Roberto Monti

monti@math.unipd.it

Erika Battaglia

erika.battaglia@unipd.it

Alberto Righini

righini@math.unipd.it

1 Dipartimento di Matematica "Tullio Levi-Civita", Università di Padova, Via Trieste 63, 35121 Padova, Italy 
The characteristic curvature $\kappa$ of a hypersurface $\Sigma \subset \mathbf{C} P^{n}$ is the curvature in direction $J N$, where $N$ is the normal to $\Sigma$ and $J$ is the complex structure of $\mathbf{C} P^{n}$, i.e., $\kappa=h(J N, J N)$ where $h$ is the second fundamental form of $\Sigma$.

For fixed $H \in \mathbb{R}$ and $n \in \mathbb{N}$, let $p(\cdot ; H, n)$ be the quadratic polynomial of the real variable $t \in \mathbb{R}$

$$
p(t ; H, n)=(2 n+1) t^{2}-2 H t-H^{2}-4\left(n^{2}-1\right) .
$$

Our first result is the following theorem.

Theorem 1.1 Let $\Sigma \subset \mathbf{C} P^{n}, n \geq 2$, be a complete connected stable hypersurface with constant $H=\operatorname{tr}(h)$. If the characteristic curvature $\kappa$ of $\Sigma$ satisfies $p(\kappa ; H, n) \geq 0$ then $\Sigma$ is a geodesic sphere of radius $r>0$ with $\tan ^{2} r=2 n+1$.

Let $\mathbf{C T} \Sigma$ be the complex tangent space of a hypersurface $\Sigma$, and let $T_{1}$ and $T_{2}$ be nonzero subbundles of $\mathbf{C} T \Sigma$ such that

$$
\mathbf{C} T \Sigma=T_{1} \oplus T_{2}
$$

and denote their dimensions by $\alpha=\operatorname{dim}_{\mathbb{R}}\left(T_{1}\right)$ and $\beta=\operatorname{dim}_{\mathbb{R}}\left(T_{2}\right)$. Then we have $\alpha+\beta=$ $\operatorname{dim}_{\mathbb{R}}(\mathbf{C} T \Sigma)=2(n-1)$ and $1 \leq \alpha<2(n-1)$. Let $h_{1}$ and $h_{2}$ be the restrictions of the second fundamental form $h$ of $\Sigma$ to $T_{1}$ and $T_{2}$, and denote by $H_{1}=\operatorname{tr}\left(h_{1}\right)$ and $H_{2}=\operatorname{tr}\left(h_{2}\right)$ their respective traces.

For fixed $H \in \mathbb{R}, n \in \mathbb{N}$ with $n \geq 2$, and $1 \leq \alpha<2(n-1)$, let $p(\cdot ; H, n, \alpha)$ be the quadratic convex polynomial of the variables $(s, t) \in \mathbb{R}^{2}$

$$
p(s, t ; H, n, \alpha)=\frac{s^{2}}{\alpha}+\frac{t^{2}}{\beta}+(s+t-H)^{2}+\frac{(s+t-2 H)^{2}}{2\left(n^{2}-1\right)}-\frac{H^{2}}{n-1}-2 n .
$$

Our second result is a refined version of Theorem 1.1.

Theorem 1.2 Let $\Sigma \subset \mathbf{C} P^{n}, n \geq 3$, be a complete connected stable hypersurface with constant $H=\operatorname{tr}(h)$. If for the decomposition (1.2), with $1 \leq \alpha=\operatorname{dim}_{\mathbb{R}}\left(T_{1}\right)<2(n-1)$, we have $p\left(H_{1}, H_{2} ; H, n, \alpha\right) \geq 0$, then $\Sigma$ is a geodesic sphere of radius $r>0$ with $\tan ^{2} r=$ $2 n+1$.

Both Theorems 1.1 and 1.2 are a consequence of the following geometric inequality that is implied by stability. Let $\nabla^{\top}$ be the Levi-Civita connection of $\mathbf{C} P^{n}$, see Sect. 3 for the notation, and consider the covariant derivative $\nabla_{J N}^{\top} N \in T \Sigma$ of the normal $N$ to $\Sigma$. We denote by $h_{N} \in \mathbf{C} T \Sigma$ the projection of $\nabla_{J N}^{\top} N$ onto $\mathbf{C} T \Sigma$. By $|h|^{2}$ we denote the squared norm of $h$.

Theorem 1.3 Let $\Sigma \subset \mathbf{C} P^{n}, n \geq 2$, be a complete stable hypersurface with constant $H=$ $\operatorname{tr}(h)$. Then we have

$$
\int_{\Sigma}\left\{|h|^{2}+\frac{(H+\kappa)^{2}+\left|h_{N}\right|^{2}}{2\left(n^{2}-1\right)}-\frac{H^{2}}{n-1}-2 n\right\} \mathrm{d} \mu \leq 0,
$$

where $\mu$ is the Riemannian hypersurface measure.

The method for obtaining formula (1.4) starts from an idea contained in the proof of [1] that geodesic spheres are the unique stable complete hypersurfaces in the standard sphere. Our first step is the isometric embedding of $\mathbf{C} P^{n}$ into $H^{n+1}$, the space of $(n+1) \times(n+1)$ 
Hermitian matrices, see [6,8]. Once the hypersurface $\Sigma$ is embedded in $H^{n+1}$, we can consider the position matrix $A \in \Sigma$ and compute its tangential Laplacian, see Theorem 4.1,

$$
\Delta A=\operatorname{tr}(\sigma)-\sigma(N, N)-\operatorname{tr}(h) N,
$$

where $\Delta$ is the Laplace-Beltrami operator of $\Sigma$ and $\sigma: T_{A} \mathbf{C} P^{n} \times T_{A} \mathbf{C} P^{n} \rightarrow T_{A}^{\perp} \mathbf{C} P^{n}$ is the second fundamental form of the immersion $\mathbf{C} P^{n} \subset H^{n+1}$. We shall make systematic use of the geometric formulas concerning $\sigma$ proved by Ros in [6,7]. They are reviewed in Sect. 3.

For a smooth function $u: \Sigma \rightarrow \mathbb{R}$, the second variation of the area functional in the normal direction $u N$ is given by the formula

$$
\mathscr{A}^{\prime \prime}(u)=-\int_{\Sigma} u \mathscr{L} u \mathrm{~d} \mu,
$$

where $\mathscr{L} u=\Delta u+\left(|h|^{2}+\operatorname{Ric}(N)\right) u$ is the Jacobi operator. When $u$ has zero mean, the deformation of $\Sigma$ encloses a region with the same volume as $\Sigma$, at the infinitesimal level.

For any fixed $V \in H^{n+1}$, the function $u_{V}=\langle\Delta A, V\rangle$ has zero mean. In Sect. 6, we compute the trace of the quadratic form $Q_{\Sigma}$ on $H^{n+1}$ defined by $Q_{\Sigma}(V)=\mathscr{A}^{\prime \prime}\left(u_{V}\right)$. If $\Sigma$ is stable, this trace is nonnegative and this fact is precisely inequality (1.4).

From (1.5), it is clear that in the computation for $\mathscr{L} u_{V}$ we need geometric formulas for $\Delta \operatorname{tr}(\sigma), \Delta \sigma(N, N)$, and $\Delta N$. The computation for $\Delta N$ is done in Theorem 4.2. The formula for $\Delta \operatorname{tr}(\sigma)$ follows easily from the formula for the trace of $\sigma$, see (3.18). The difficult task is to compute the tangential Laplacian of $\sigma(N, N)$. This is done in Sect. 5, and the resulting formula is in (5.4). In Sect. 6, we collect all these preliminary computations and we finish the proof of Theorem 1.3.

The proof of Theorem 1.1 now follows from Theorem 1.3 using Takagi's and CecilRyan's characterization of the sphere in $\mathbf{C} P^{n}$ as the unique hypersurface having precisely two (constant) different curvatures. The proof of Theorem 1.2 uses Takagi's classification of hypersurfaces in $\mathbf{C} P^{n}, n \geq 3$, having precisely three different constant curvatures: they are either geodesics tubes around $\mathbf{C} P^{k}$ for some $k=1, \ldots, n-1$ or geodesics tubes around the real projective space $\mathbf{R} P^{n}$. The details are in the final Sect. 7, while the preliminary results on spheres and tubes are reviewed in Sect. 2.

In this paper, by "hypersurface" we always mean "embedded hypersurface" in $\mathbf{C} P^{n}$.

\section{Geometry of spheres and tubes}

The $n$-dimensional complex projective space is the quotient of the unit sphere $\mathbf{S}^{2 n+1}=\{z \in$ $\left.\mathbf{C}^{n+1}:|z|=1\right\}$ by the Hopf action of $\mathbf{S}^{1},\left(\mathrm{e}^{i \vartheta}, z\right) \mapsto \mathrm{e}^{i \vartheta} z$. We denote by $[z]$ the equivalence class of $z \in \mathbf{S}^{2 n+1}$. The tangent space of $\mathbf{C} P^{n}$ at the point $[z]$ is

$$
T_{[z]} \mathbf{C} P^{n}=\left\{w \in \mathbf{C}^{n+1}: z \cdot \bar{w}=0\right\},
$$

where $z \cdot \bar{w}=z_{1} \bar{w}_{1}+\cdots+z_{n+1} \bar{w}_{n+1}$ is the standard Hermitian product of $\mathbf{C}^{n+1}$. The complex structure on $T_{[z]} \mathbf{C} P^{n}$ is given by $J w=i w$, the standard multiplication by $i$ of $w \in T_{[z]} \mathbf{C} P^{n} \subset \mathbf{C}^{n+1}$.

The metric $\langle\zeta, w\rangle_{F S}=\operatorname{Re}(\zeta \cdot \bar{w})$, with $\zeta, w \in T_{[z]} \mathbf{C} P^{n}$, is the Fubini-Study metric of $\mathbf{C} P^{n}$, that makes the complex projective space a Riemannian manifold. The induced distance function $d: \mathbf{C} P^{n} \times \mathbf{C} P^{n} \rightarrow[0, \pi / 2]$ is $d([z],[w])=\arccos |z \cdot \bar{w}|$.

Let $\Sigma \subset \mathbf{C} P^{n}$ by a $C^{\infty}$-smooth hypersurface oriented by the unit normal $N$. We define the second fundamental form $h$ of $\Sigma$ with the following sign convention 


$$
h(X, Y)=\left\langle\nabla_{X}^{\top} N, Y\right\rangle, \quad X, Y \in T_{[z]} \Sigma .
$$

The characteristic curvature of $\Sigma$ at the point $[z] \in \Sigma$ is $\kappa=h(J N, J N)$.

For any fixed $[w] \in \mathbf{C} P^{n}$ and $0<r<\pi / 2$, the geodesic sphere centered at $[w]$ with radius $r$ is

$$
\Sigma_{r}=\left\{[z] \in \mathbf{C} P^{n}:|z \cdot \bar{w}|=\cos r\right\} .
$$

We omit reference to the center. The curvatures of $\Sigma_{r}$ are well known, see, e.g., [3, Example 1 page 493]. Letting $t=\tan r$, they are

$$
\begin{aligned}
& \lambda=\cot r=\frac{1}{t}, \quad \text { with multiplicity } 2(n-1), \\
& \kappa=2 \cot (2 r)=\frac{1}{t}-t, \quad \text { the characteristic curvature. }
\end{aligned}
$$

These two curvatures are constant and distinct for each value of $t>0$. In Takagi [9] proved that this property characterizes the sphere.

Proposition 2.1 If $\Sigma \subset \mathbf{C} P^{n}, n \geq 2$, is a connected hypersurface with precisely two distinct constant curvatures, then $\Sigma$ is a subset of a sphere $\Sigma_{r}$.

In fact, the constancy assumption on the curvatures can be dropped, see [3].

We now discuss tubes around $\mathbf{C} P^{k}$. For $k=1, \ldots, n-1$, the natural inclusion $\mathbf{S}^{2 k+1}=$ $\left\{z \in \mathbf{S}^{2 n+1}: z_{k+2}=\cdots=z_{n+1}=0\right\} \subset \mathbf{S}^{2 n+1}$ induces the inclusion $\mathbf{C} P^{k} \subset \mathbf{C} P^{n}$. For $0<r<\pi / 2$, we define the tube

$$
\begin{aligned}
T_{r}^{k} & =\left\{[z] \in \mathbf{C} P^{n}: \operatorname{dist}\left([z], \mathbf{C} P^{k}\right)=r\right\} \\
& =\left\{[z]=\left[\left(z^{\prime}, z^{\prime \prime}\right)\right] \in \mathbf{C} P^{n}:|z|=1, z^{\prime} \in \mathbf{C}^{k+1},\left|z^{\prime}\right|=\cos r\right\} .
\end{aligned}
$$

The curvatures of $T_{r}^{k}$ are computed in [3]. Letting $t=\tan r$, they are

$$
\begin{aligned}
& \lambda_{1}=\cot \left(r-\frac{\pi}{2}\right)=-t, \quad \text { with multiplicity } 2 k, \\
& \lambda_{2}=\cot r=\frac{1}{t}, \quad \text { with multiplicity } 2 \ell=2(n-1-k), \\
& \kappa=2 \cot (2 r)=\frac{1}{t}-t, \quad \text { the characteristic curvature. }
\end{aligned}
$$

These three curvatures are constant and distinct for each value of $t>0$. In particular, $T_{r}^{k}$ has constant mean curvature. For $r+s=\pi / 2$ and $k+\ell=n-1$ the hypersurfaces $T_{r}^{k}$ and $T_{s}^{\ell}$ are congruent.

The tubes $T_{r}^{k}$ share with the sphere $\Sigma_{r}$ the property of being stable for some value of $r$. The following theorem is proved in [2, Theorem 1.3] using the method of $G$-stability.

Theorem 2.2 Let $n \geq 2$ and $k=1, \ldots, n-1$. Then:

(1) The sphere $\Sigma_{r}$ is stable if and only if $\tan ^{2} r \leq 2 n+1$.

(2) The tube $T_{r}^{k}$ is stable if and only if

$$
\frac{2 n-2 k-1}{2 k+3} \leq \tan ^{2} r \leq \frac{2 n-2 k+1}{2 k+1} .
$$

Finally, we consider geodesics tubes around $\mathbf{R} P^{n}$. We start from the following embedding of the sphere $\mathbf{S}^{n}$ into $\mathbf{S}^{2 n+1}$ :

$$
\mathbf{S}^{n}=\left\{z \in \mathbf{C}^{n+1}:|z|=1, z=\bar{z}\right\} \subset \mathbf{S}^{2 n+1} .
$$


Passing to the quotient, the inclusion $\mathbf{S}^{n} \subset \mathbf{S}^{2 n+1}$ gives an embedding of $\mathbf{R} P^{n}$ into $\mathbf{C} P^{n}$. The distance of $z \in \mathbf{S}^{2 n+1}$ from $\mathbf{S}^{n}$ is

$$
\operatorname{dist}\left(z, \mathbf{S}^{n}\right)=\frac{1}{2} \arccos \left(\left|\sum_{j=1}^{n+1} z_{j}^{2}\right|\right),
$$

and it does not depend on the equivalence class of $z$. The level sets of this distance form the isoparametric family of hypersurfaces in $\mathbf{S}^{2 n+1}$ studied in [5, Theorem 1]. Hence, the geodesic tube in $\mathbf{C} P^{n}$ with radius $r$ around $\mathbf{R} P^{n}$ is

$$
V_{r}=\left\{[z] \in \mathbf{C} P^{n}:\left|\sum_{j=1}^{n+1} z_{j}^{2}\right|=\cos 2 r\right\} .
$$

The curvatures of $V_{r}$ are computed in [3] starting from the formulas in [5] for the preimage of $V_{r}$ in $\mathbf{S}^{2 n+1}$. Letting $t=\tan r$, they are

$$
\begin{aligned}
\lambda_{1} & =-\cot r=-\frac{1}{t}, \quad \text { with multiplicity } n-1, \\
\lambda_{2} & =-\cot \left(\frac{\pi}{2}-r\right)=t, \quad \text { with multiplicity } n-1, \\
\kappa & =2 \cot \left(\frac{\pi}{2}-2 r\right)=\frac{4 t}{1-t^{2}}, \quad \text { the characteristic curvature. }
\end{aligned}
$$

These three curvatures are constant and distinct for each value of $t \in(0,1)$. We have a third example of a complete constant mean curvature hypersurface in $\mathbf{C} P^{n}$. We will see in Lemma 6.5 that $V_{r}$ is not stable for any $0<r<\pi / 4$.

Takagi [10] proved that the tubes $V_{r}$ and the tubes $T_{r}^{k}$ are characterized by the fact of having precisely three distinct and constant curvatures.

Proposition 2.3 Let $n \geq 3$. If $\Sigma \subset \mathbf{C} P^{n}$ is a connected hypersurface with three distinct constant curvatures, then $\Sigma$ is a subset of some tube $T_{r}^{k}$ or $V_{r}$.

We shall use Proposition 2.3 and Theorem 2.2 in the proof of Theorem 1.2 in Sect. 7.

\section{Geometry of the isometric immersion of $\mathrm{CP}^{n}$ into $\mathrm{H}^{\mathrm{n}+1}$}

Let $H^{n+1}=\left\{A \in \operatorname{gl}(n+1, \mathbf{C}): \bar{A}=A^{t}\right\}$ be the set of $(n+1)$-dimensional Hermitian matrices. This is a $(n+1)^{2}$-dimensional real subspace of $\mathrm{gl}(n+1, \mathbf{C})$. The standard scalar product on $H^{n+1}$ is

$$
\langle A, B\rangle:=\frac{1}{2} \operatorname{tr}(A B), \quad A, B \in H^{n+1} .
$$

Let $\Phi: \mathbf{C} P^{n} \rightarrow H^{n+1}$ be the mapping that takes the equivalence class $[z] \in \mathbf{C} P^{n}$ to the Hermitian matrix $A=\Phi([z]) \in H^{n+1}$ of the projection of $\mathbf{C}^{n+1}$ onto the complex line [z]. The matrix $A$ satisfies $A^{2}=A$ because it is a projection and $\operatorname{tr}(A)=1$, because it projects onto a complex line. It can be checked that $\Phi$ is an isometry from $\mathbf{C} P^{n}$ with the Fubini-Study metric into $H^{n+1}$ with the metric (3.1). Hence, from now on we identify the complex projective space with

$$
\mathbf{C} P^{n}=\left\{A \in H^{n+1}: A^{2}=A, \operatorname{tr}(A)=1\right\} .
$$


For details on this identification and for the proof of the following lemmas, we refer the reader to [6]. Our normalization in (3.1) of the scalar product is different from the one by Ros. Namely, the relation between the metric $g$ used by Ross and the metric in (3.1) is $g(A, B)=4\langle A, B\rangle$. The isometric embedding $\Phi$ was introduced in [8].

For any $A \in \mathbf{C} P^{n}$, we denote by $T_{A} \mathbf{C} P^{n}$ and $T_{A}^{\perp} \mathbf{C} P^{n}$ the tangent space and the normal space of $\mathbf{C} P^{n}$ at the point $A \in H^{n+1}$, respectively.

Lemma 3.1 For any $A \in \mathbf{C} P^{n}$, we have:

$$
\begin{aligned}
T_{A} \mathbf{C} P^{n} & =\left\{X \in H^{n+1}: X A+A X=X\right\}, \\
T_{A}^{\perp} \mathbf{C} P^{n} & =\left\{Z \in H^{n+1}: A Z=Z A\right\} .
\end{aligned}
$$

For the proof see [6]. We easily see that $A, I \in T_{A}^{\perp} \mathbf{C} P^{n}$, where $I$ is the identity matrix. We call the matrix $A_{0} \in \mathbf{C} P^{n}$

$$
A_{0}:=\left(\begin{array}{ll}
1 & 0 \\
0 & 0
\end{array}\right)
$$

the origin of $\mathbf{C} P^{n}$. This is the projection onto the complex line of the versor $\mathrm{e}_{0}=$ $(1,0, \ldots, 0) \in \mathbf{C}^{n+1}$.

For $i, j \in\{0,1, \ldots, n\}$, let $E_{i j}$ be the $(n+1) \times(n+1)$ matrix with entry 1 at the position $(i, j)$ and with 0 elsewhere. With this notation we have $E_{00}=A_{0}$. Then the matrices $X_{1}, \ldots, X_{n}, \widehat{X}_{1}, \ldots, \widehat{X}_{n}$, where for $j=1, \ldots, n$

$$
X_{j}=E_{j 0}+E_{0 j} \quad \text { and } \quad \widehat{X}_{j}=i E_{j 0}-i E_{0 j},
$$

form an orthonormal basis for the tangent space of $\mathbf{C} P^{n}$ at the point $A_{0}$. The identity $\widehat{X}_{j}=$ $J X_{j}$ can be checked using formula (3.10) below for the complex structure.

For any point $A \in \mathbf{C} P^{n}$, there exists a (non-unique) unitary transformation $Q \in U(n+1)$ such that $A=Q A_{0} Q^{-1}$. The conjugation $T_{Q}: H^{n+1} \rightarrow H^{n+1}, T_{Q} X=Q X Q^{-1}$, preserves the metric of $H^{n+1}$ :

$$
\left\langle T_{Q} X, T_{Q} Y\right\rangle=\frac{1}{2} \operatorname{tr}\left(Q X Y Q^{-1}\right)=\frac{1}{2} \operatorname{tr}(X Y)=\langle X, Y\rangle, \quad X, Y \in H^{n+1} .
$$

In particular, $T_{Q}$ maps isometrically the tangent space $T_{A_{0}} \mathbf{C} P^{n}$ onto $T_{A} \mathbf{C} P^{n}$. We will use these isometries to reduce computations of isometric-invariant quantities to the origin $A_{0}$.

By elementary computations based on the projection equation $A^{2}=A$ and on the equation $X=A X+X A$ for tangent vectors, it is possible to check the following algebraic identities.

Lemma 3.2 For any point $A \in \mathbf{C} P^{n}$ and for any vector fields $X, Y \in T_{A} \mathbf{C} P^{n}$, we have:

$$
\begin{aligned}
& A X Y=X Y A, \\
& A X A=0, \\
& X(I-2 A)=-(I-2 A) X, \\
& (I-2 A)^{2}=I, \\
& (I-2 A) X Y=X Y(I-2 A) .
\end{aligned}
$$

Using the isometric identification $\Phi$, the natural complex structure on $T_{[z]} \mathbf{C} P^{n} \subset \mathbf{C}^{n+1}$ can be taken to $T_{A} \mathbf{C} P^{n} \subset H^{n+1}$. The resulting mapping is described in the following proposition. 
Proposition 3.3 For any $A \in \mathbf{C} P^{n}$, the mapping $J_{A}: T_{A} \mathbf{C} P^{n} \rightarrow T_{A} \mathbf{C} P^{n}$ defined by the formula

$$
J_{A} X=i(I-2 A) X,
$$

satisfies the following properties:

(i) it is an isometry;

(ii) it satisfies $J_{A}^{2}=-\mathrm{Id}$, where Id is the identity mapping;

(iii) it commutes with the isometries $T_{Q}$, i.e., for any $A, B \in \mathbf{C} P^{n}$ with $A=T_{Q} B$ for some $Q \in U(n+1)$, and for any $X \in T_{B} \mathbf{C} P^{n}$, we have

$$
J_{A} T_{Q} X=T_{Q} J_{B} X .
$$

We compute the mean curvature of the immersion of $\mathbf{C} P^{n}$ into $H^{n+1}$. For any $A \in \mathbf{C} P^{n}$, we define the orthogonal projections $\pi_{A}^{\top}: H^{n+1} \rightarrow T_{A} \mathbf{C} P^{n}$ and $\pi_{A}^{\perp}: H^{n+1} \rightarrow T_{A}^{\perp} \mathbf{C} P^{n}$. Explicit formulas for $\pi^{\top}$ and $\pi^{\perp}$ can be expressed using the symmetric product $\pi: H^{n+1} \times$ $H^{n+1} \rightarrow H^{n+1}$

$$
\pi(X, Y)=X Y+Y X .
$$

Notice that by (3.3) and (3.5), we have $\pi: T_{A} \mathbf{C} P^{n} \times T_{A} \mathbf{C} P^{n} \rightarrow T_{A}^{\perp} \mathbf{C} P^{n}$.

Lemma 3.4 For any $A \in \mathbf{C} P^{n}$ and $X \in H^{n+1}$, we have

$$
\begin{aligned}
& \pi_{A}^{\top}(X)=\pi(A, X)-2 A X A, \\
& \pi_{A}^{\perp}(X)=X-\pi(A, X)+2 A X A .
\end{aligned}
$$

Proof The mapping defined by formula (3.12) is linear and is the identity on $T_{A} \mathbf{C} P^{n}$. Indeed, for any $X \in T_{A} \mathbf{C} P^{n}$, by (3.6) and (3.2) we have $\pi_{A}^{\top}(X)=A X+X A=X$. We claim that $\pi_{A}^{\top}(X) \in T_{A} \mathbf{C} P^{n}$ for any $X \in H^{n+1}$. Indeed, we have

$$
\begin{aligned}
\pi_{A}^{\top}(X) A+A \pi_{A}^{\top}(X) & =A X A+X A-2 A X A+A X+A X A-2 A X A \\
& =\pi(A, X)-2 A X A=\pi_{A}^{\top}(X),
\end{aligned}
$$

where we used $A^{2}=A$.

Then formula (3.12) defines the projection onto $T_{A} \mathbf{C} P^{n}$. Formula (3.13) follows from (3.12).

We split the standard connection $\nabla$ of $H^{n+1}$ into the part that is tangent to $\mathbf{C} P^{n}$ and the part that is normal. Namely, for $X \in \Gamma\left(T \mathbf{C} P^{n}\right), Y \in \Gamma\left(T H^{n+1}\right)$, and $A \in \mathbf{C} P^{n}$ we let $\nabla_{X}^{\top} Y(A)=\pi_{A}^{\top}\left(\nabla_{X} Y\right)$, and $\nabla_{X}^{\perp} Y(A)=\pi_{A}^{\perp}\left(\nabla_{X} Y\right)$. By (3.12) and (3.13), we have the formulas

$$
\begin{aligned}
& \nabla_{X}^{\top} Y=\pi\left(A, \nabla_{X} Y\right)-2 A\left(\nabla_{X} Y\right) A, \\
& \nabla_{X}^{\perp} Y=\nabla_{X} Y-\pi\left(A, \nabla_{X} Y\right)+2 A\left(\nabla_{X} Y\right) A .
\end{aligned}
$$

The second fundamental form of the immersion of $\mathbf{C} P^{n}$ into $H^{n+1}$ is the mapping $\sigma_{A}$ : $T_{A} \mathbf{C} P^{n} \times T_{A} \mathbf{C} P^{n} \rightarrow T_{A}^{\perp} \mathbf{C} P^{n}, A \in \mathbf{C} P^{n}$, defined by $\sigma_{A}(X, Y)=\nabla_{X}^{\perp} Y(A)$. When no confusion arises, we drop the subscript $A$ and write $\sigma=\sigma_{A}$.

The non-normalized mean curvature vector of the immersion is the trace of $\sigma$, i.e.,

$$
\mathscr{H}=\operatorname{tr}(\sigma)=\sum_{i=1}^{2 n} \sigma\left(X_{i}, X_{i}\right),
$$

where $X_{1}, \ldots, X_{2 n}$ is any orthonormal frame of $T_{A} \mathbf{C} P^{n}$. 
Proposition 3.5 For any $A \in \mathbf{C} P^{n}$ and $X, Y \in T_{A} \mathbf{C} P^{n}$, we have

$$
\sigma(X, Y)=\pi(X, Y)(I-2 A),
$$

and the trace of $\sigma$ is

$$
\mathscr{H}=4(I-(n+1) A), \quad A \in \mathbf{C} P^{n} .
$$

For a proof of (3.17) and (3.18), see [6, Proposition 1.4].

Remark 3.6 Using (3.17) and (3.10), it is possible to check the following identity for any $X, Y, V, W \in T_{A} \mathbf{C} P^{n}$ :

$$
\begin{aligned}
\langle\sigma(X, Y), \sigma(V, W)\rangle= & 2\langle X, Y\rangle\langle V, W\rangle+\langle X, W\rangle\langle Y, V\rangle+\langle X, V\rangle\langle Y, W\rangle \\
& +\langle X, J W\rangle\langle Y, J V\rangle+\langle X, J V\rangle\langle Y, J W\rangle .
\end{aligned}
$$

The Weingarten endomorphism of the immersion is the mapping $\Lambda: T_{A} \mathbf{C} P^{n} \times T_{A}^{\perp} \mathbf{C} P^{n} \rightarrow$ $T_{A} \mathbf{C} P^{n}, A \in \mathbf{C} P^{n}$, defined by the formula $\Lambda(X, Z)=\Lambda_{Z}(X)=-\nabla_{X}^{\top} Z$.

Proposition 3.7 For any $A \in \mathbf{C} P^{n}, X \in T_{A} \mathbf{C} P^{n}$, and $Z \in T_{A}^{\perp} \mathbf{C} P^{n}$, we have

$$
\Lambda_{Z}(X)=(X Z-Z X)(I-2 A) \text {. }
$$

For the proof see [6, Proposition 1.4]. We establish some identities linking $\Lambda$ and $\sigma$. Let $X_{1}, \ldots, X_{2 n}$ be an orthonormal frame for $T \mathbf{C} P^{n}$ and we use the notation $N=X_{2 n}$. In the sequel, we also let

$$
\begin{aligned}
\pi_{i j}=\pi\left(X_{i}, X_{j}\right) & \text { and } \quad \pi_{i, N}=\pi\left(X_{i}, N\right), \\
\sigma_{i j}=\sigma\left(X_{i}, X_{j}\right) & \text { and } \quad \sigma_{i, N}=\sigma\left(X_{i}, N\right) .
\end{aligned}
$$

The second fundamental form $\sigma(X, Y)$ is defined when $X$ and $Y$ are tangent sections of $\mathbf{C} P^{n}$. However, the right-hand side of (3.17) is defined for any $X, Y \in H^{n+1}$. In the next lemma and in the next sections, we will use (3.17) as the general definition of $\sigma$.

Lemma 3.8 Let $X_{1}, \ldots, X_{2 n-1}, N$ be an orthonormal frame of $\mathbf{C} P^{n}$. Then for any $i, j=$ $1, \ldots, 2 n-1$ we have

$$
\Lambda_{\sigma_{j, N}}\left(X_{i}\right)=\pi\left(\pi_{j, N}, X_{i}\right)=2 \pi_{j, N} X_{i}-\sigma\left(\sigma_{i j}, N\right)-\sigma\left(X_{j}, \sigma_{i, N}\right) .
$$

Proof We prove the identity on the left of (3.21). By (3.17), (3.20), (3.7), and (3.8), we get

$$
\begin{aligned}
\Lambda_{\sigma_{j, N}}\left(X_{i}\right) & =\left(X_{i} \sigma_{j, N}-\sigma_{j, N} X_{i}\right)(I-2 A) \\
& =\left(X_{i} \pi_{j, N}(I-2 A)-\pi_{j, N}(I-2 A) X_{i}\right)(I-2 A) \\
& =X_{i} \pi_{j, N}+\pi_{j, N} X_{i}=\pi\left(\pi_{j, N}, X_{i}\right) .
\end{aligned}
$$

Now we check the identity on the right. Using (3.7) and (3.8), we have:

$$
\begin{aligned}
2 \pi_{j, N} X_{i}- & {\left[\pi\left(\sigma_{i j}, N\right)+\pi\left(X_{j}, \sigma_{i, N}\right)\right](I-2 A) } \\
= & -\left(\pi_{i j}(I-2 A) N+N \pi_{i j}(I-2 A)+X_{j} \pi_{i, N}(I-2 A)\right. \\
& \left.+\pi_{i, N}(I-2 A) X_{j}\right)(I-2 A)+2 \pi_{j, N} X_{i} \\
= & \pi_{i j} N-N \pi_{i j}-X_{j} \pi_{i, N}+\pi_{i, N} X_{j}+2 \pi_{j, N} X_{i} \\
= & X_{i} X_{j} N+N X_{j} X_{i}+X_{j} N X_{i}+X_{i} N X_{j} \\
= & X_{i} \pi_{j, N}+\pi_{j, N} X_{i} \\
= & \pi\left(X_{i}, \pi_{j, N}\right) .
\end{aligned}
$$


Lemma 3.9 For any orthonormal frame $X_{1}, \ldots, X_{2 n-1}, N$ of $\mathbf{C} P^{n}$, we have

$$
\sum_{i=1}^{2 n-1} \Lambda_{\sigma_{i, N}}\left(X_{i}\right)=2(n-1) N .
$$

Proof It is enough to verify (3.23) at the point $A_{0} \in \mathbf{C} P^{n}$, with the frame (3.4) where $N=\widehat{X}_{n}$. Using formula (3.20) for $\Lambda$ and the identities (3.7) and (3.8), for any $i=1, \ldots, n$ and $j=1, \ldots, n-1$ we find

$$
\begin{aligned}
& \Lambda_{\sigma_{i, N}}\left(X_{i}\right)=\delta_{i n}\left(N-2 \widehat{X}_{i}\right)+N, \\
& \Lambda_{\sigma_{\widehat{j}, N}}\left(\widehat{X}_{j}\right)=\delta_{j n}\left(2 \widehat{X}_{j}+N\right)+N=N .
\end{aligned}
$$

Summing up, we obtain (3.23).

\section{Laplacian of position and normal}

Let $\Sigma \subset \mathbf{C} P^{n}$ be a hypersurface oriented by the unit normal $N$. In the following we adopt the short notation $\sigma_{N}=\sigma(N, N)$ and $\pi_{N}=\pi(N, N)$. In this section, we compute $\Delta A$ and $\Delta N$, where $\Delta$ is the Laplace-Beltrami operator of $\Sigma$.

The second fundamental form of $\Sigma$ is the mapping $h: T_{A} \Sigma \times T_{A} \Sigma \rightarrow \mathbb{R}, h(X, Y)=$ $\left\langle\nabla_{X}^{\top} N, Y\right\rangle$, and we denote its trace by $H=\operatorname{tr}(h)$.

Notation From now on, we will omit the symbol of sum over repeated indices. The repeated index always runs from 1 to $2 n-1$. In the other cases, we will write the sum.

Theorem 4.1 Let $\Sigma \subset \mathbf{C} P^{n}$ be an oriented hypersurface with normal $N$. The position matrix A satisfies the equation

$$
\Delta A=\operatorname{tr}(\sigma)-\sigma_{N}-\operatorname{tr}(h) N, \quad A \in \Sigma,
$$

where $\sigma$ is the second fundamental form of the immersion $\mathbf{C} P^{n} \subset H^{n+1}$.

Proof Without loss of generality, we can assume that $A_{0} \in \Sigma$ and we check formula (4.1) at the point $A_{0}$. Let $X_{1}, \ldots, X_{2 n-1}$ be a frame of vector fields tangent to $\Sigma$ given by normal coordinates at $A_{0}$. Namely, for all $i, j=1, \ldots, 2 n-1$, we have

$$
\nabla_{X_{i}}^{\Sigma} X_{j}\left(A_{0}\right)=0 \text {. }
$$

We are denoting by $\nabla^{\Sigma}$ the Levi-Civita connection of $\Sigma$. This is the restriction of $\nabla^{\top}$ to $\Sigma$, projected onto $T \Sigma$.

In the next line and in the rest of the paper, we shall use the identity

$$
\nabla_{X} A=X \text {. }
$$

With sum over repeated indices for $j=1, \ldots, 2 n-1$, we have

$$
\begin{aligned}
\left.\Delta A\right|_{A=A_{0}} & =\left.\nabla_{X_{j}} \nabla_{X_{j}} A\right|_{A=A_{0}}=\nabla_{X_{j}} X_{j}\left(A_{0}\right) \\
& =\nabla_{X_{j}}^{\top} X_{j}\left(A_{0}\right)+\sigma_{A_{0}}\left(X_{j}, X_{j}\right) .
\end{aligned}
$$

In the last equality, we used the definition of the second fundamental form $\sigma$. Again in $A_{0}$, by (4.2) we obtain

$$
\nabla_{X_{j}}^{\top} X_{j}=\left\langle\nabla_{X_{j}}^{\top} X_{j}, N\right\rangle N=-\left\langle X_{j}, \nabla_{X_{j}}^{\top} N\right\rangle N=-\operatorname{tr}(h) N .
$$


Since $X_{1}, \ldots, X_{2 n-1}, N$ is an orthonormal frame of $\mathbf{C} P^{n}$, from Definition (3.16) of $\mathscr{H}$ we have $\sigma\left(X_{j}, X_{j}\right)=\mathscr{H}-\sigma_{N}$, and this ends the proof.

In the next theorem, we compute a formula for $\Delta N$. The second fundamental form $h$ of $\Sigma$ can be identified with a linear operator on $T_{A} \Sigma$. The restriction of $\sigma$ to $T_{A} \Sigma$ can be identified with a linear operator from $T_{A} \Sigma$ to $\operatorname{End}\left(T_{A} \Sigma, T_{A}^{\perp} \mathbf{C} P^{n}\right)$. Hence, the composition $\sigma h=\sigma \circ h$ is a linear operator from $T_{A} \Sigma$ to $\operatorname{End}\left(T_{A} \Sigma, T_{A}^{\perp} \mathbf{C} P^{n}\right)$. Namely, for any $X, Y \in T_{A} \Sigma$ we have $\sigma h(X)[Y]=\sigma(h(X), Y)$. We denote its trace by

$$
\operatorname{tr}(\sigma h)=\sigma h\left(X_{i}\right)\left[X_{i}\right]=\sigma\left(h_{i j} X_{j}, X_{i}\right)=h_{i j} \sigma_{i j} \in T_{A}^{\perp} \mathbf{C} P^{n},
$$

where $\sigma_{i j}=\sigma\left(X_{i}, X_{j}\right)$ and $h_{i j}=h\left(X_{i}, X_{j}\right)$ for any orthonormal frame $X_{1}, \ldots, X_{2 n-1}$ of $T_{A} \Sigma$.

Theorem 4.2 Let $\Sigma \subset \mathbf{C} P^{n}$ be an oriented hypersurface with constant mean curvature. The normal $N$ to the hypersurface satisfies the equation

$$
\Delta N=2 \operatorname{tr}(\sigma h)-\left(|h|^{2}+2(n-1)\right) N-\operatorname{tr}(h) \sigma_{N} .
$$

The proof is preceded by a numbers of lemmas. We are using a frame of vector fields satisfying (4.2).

Lemma 4.3 Let $\Sigma \subset \mathbf{C} P^{n}$ be an orientable hypersurface with constant mean curvature. At the center $A_{0} \in \Sigma$ of normal coordinates, the entries of the second fundamental form $h_{i j}=h\left(X_{i}, X_{j}\right)$ satisfy for each $j=1, \ldots, 2 n-1$ the equations

$$
X_{i} h_{i j}=0
$$

with sum over the repeated index.

Proof By the Codazzi's equations, we have the identities for $i, j, \ell=1, \ldots, 2 n-1$

$$
\begin{aligned}
X_{j} h_{i \ell}-X_{i} h_{j \ell}= & \left\langle R\left(X_{i}, X_{j}\right) N, X_{\ell}\right\rangle+\left\langle\nabla_{X_{j}}^{\top} N, \nabla_{X_{i}}^{\top} X_{\ell}\right\rangle-\left\langle\nabla_{X_{i}}^{\top} N, \nabla_{X_{j}}^{\top} X_{\ell}\right\rangle+ \\
& -\left\langle N, \nabla_{\left[X_{i}, X_{j}\right]}^{\top} X_{\ell}\right\rangle,
\end{aligned}
$$

where $N$ is the normal to $\Sigma$ and $R$ is the Riemann curvature tensor of $\mathbf{C} P^{n}$. Since $\nabla^{\top}$ has vanishing torsion, at the point $A_{0}$ we have by (4.2)

$$
\left[X_{i}, X_{j}\right]=\nabla_{X_{i}}^{\top} X_{j}-\nabla_{X_{j}}^{\top} X_{i}=0 .
$$

Thus, at the point $A_{0}$ the previous identity reduces to

$$
X_{j} h_{i \ell}-X_{i} h_{j \ell}=\left\langle R\left(X_{i}, X_{j}\right) N, X_{\ell}\right\rangle
$$

Letting $i=\ell$, summing up in $i=1, \ldots, 2 n-1$, and using the fact that $\Sigma$ has constant mean curvature, we obtain

$$
X_{i} h_{i j}=-\left\langle R\left(X_{i}, X_{j}\right) N, X_{i}\right\rangle=-\operatorname{Ric}\left(X_{j}, N\right) .
$$

The last equality follows from the standard symmetries of the curvature operator. In fact, we have $\left\langle R\left(N, X_{j}\right) N, N\right\rangle=\left\langle R\left(X_{j}, N\right) N, N\right\rangle=-\left\langle R\left(N, X_{j}\right) N, N\right\rangle$ and thus $\left\langle R\left(N, X_{j}\right) N, N\right\rangle=0$. The complex projective space is a Kähler manifold, and thus, it is an Einstein manifold. From the orthogonality of $X_{j}$ and $N$, it follows that $\operatorname{Ric}\left(X_{j}, N\right)=0$. 
Lemma 4.4 Let $\Sigma \subset \mathbf{C} P^{n}$ be an orientable hypersurface with normal $N$ and $H=\operatorname{tr}(h)$. At the center of normal coordinates, we have the identity

$$
\nabla_{X_{i}} \sigma_{i, N}=\operatorname{tr}(\sigma h)-2(n-1) N-H \sigma_{N} .
$$

Proof By (3.17), (3.20) and the definition of second fundamental form $\sigma$, we have

$$
\begin{aligned}
\nabla_{X_{i}} \sigma_{i, N} & =\nabla_{X_{i}} \pi_{i, N}(I-2 A)+\pi_{i, N} \nabla_{X_{i}}(I-2 A) \\
& =\sigma\left(\nabla_{X_{i}} X_{i}, N\right)+\sigma\left(X_{i}, \nabla_{X_{i}} N\right)-2 \pi_{i, N} X_{i} \\
& =-h_{i i} \sigma_{N}+\sigma\left(\sigma_{i i}, N\right)+h_{i j} \sigma_{i j}+\sigma\left(X_{i}, \sigma_{i, N}\right)-2 \pi_{i, N} X_{i} . \\
& =-h_{i i} \sigma_{N}+h_{i j} \sigma_{i j}+(*),
\end{aligned}
$$

where $(*)=\sigma\left(\sigma_{i i}, N\right)+\sigma\left(X_{i}, \sigma_{i, N}\right)-2 \pi_{i, N} X_{i}$. Using (3.21) in the particular case when $i=j$, we deduce that $(*)=-\Lambda_{\sigma_{i, N}}\left(X_{i}\right)$.

Hence, we proved that

$$
\nabla_{X_{i}} \sigma_{i, N}=-h_{i i} \sigma_{N}+h_{i j} \sigma_{i j}-\Lambda_{\sigma_{i, N}}\left(X_{i}\right),
$$

and our claim (4.5) follows from (3.23).

Remark 4.5 Starting from

$$
\nabla_{X_{i}} \sigma_{i, N}=\nabla_{X_{i}}^{\top} \sigma_{i, N}+\nabla_{X_{i}}^{\perp} \sigma_{i, N}=-\Lambda_{\sigma_{i, N}}\left(X_{i}\right)+\nabla_{X_{i}}^{\perp} \sigma_{i, N},
$$

and using (4.6), we obtain

$$
\nabla_{X_{i}}^{\perp} \sigma_{i, N}=-H \sigma_{N}+h_{i j} \sigma_{i j} .
$$

Proof of Theorem 4.2 We check formula (4.3) using normal coordinates at the point $A_{0} \in \Sigma$. Using $|N|^{2}=1$ and $\nabla_{X_{i}}^{\perp} N=\sigma\left(X_{i}, N\right)$ we obtain

$$
\Delta N=\nabla_{X_{i}} \nabla_{X_{i}} N=\nabla_{X_{i}}\left(\nabla_{X_{i}}^{\perp} N+\nabla_{X_{i}}^{\top} N\right)=\nabla_{X_{i}}\left\{\sigma_{i, N}+h_{i j} X_{j}\right\} .
$$

From (4.4) and (4.2), we deduce that, at the point $A_{0}$,

$$
\nabla_{X_{i}}\left(h_{i j} X_{j}\right)=h_{i j} \nabla_{X_{i}} X_{j}=h_{i j}\left\{\sigma_{i j}-h_{i j} N\right\}=-|h|^{2} N+h_{i j} \sigma_{i j} .
$$

By (4.5) we have

$$
\nabla_{X_{i}} \sigma_{i, N}=-H \sigma_{N}+h_{i j} \sigma_{i j}-2(n-1) N,
$$

and then $\Delta N=-\left(|h|^{2}+2(n-1)\right) N+2 h_{i j} \sigma_{i j}-H \sigma_{N}$.

\section{Laplacian of $\sigma_{N}$}

Let $A$ be the position matrix. In the second variation of the area of a hypersurface $\Sigma$, we shall use normal deformations associated with the coordinate functions of the matrix $u=\Delta A$. In our formula (4.1) for $\Delta A$, there is the term $\sigma_{N}$ and thus to know $\Delta u$ we need $\Delta \sigma_{N}$. In this section we compute this crucial quantity.

Let $X_{1}, \ldots, X_{2 n-1}$ be an orthonormal frame of $T \Sigma$ such that $X_{n+j}=J X_{j}$ for all $j=1, \ldots, n-1$. Then we have $X_{n}=-J N$, where $N$ is the normal to $\Sigma$. The tangent vector

$$
h_{N}=\sum_{j=1, j \neq n}^{2 n-1} h\left(J N, X_{j}\right) X_{j} \in \mathbf{C T} \Sigma
$$


does not depend on the given frame. The vector $h_{N}$ is the projection onto $\mathbf{C} T \Sigma$ of $\nabla_{J N}^{\top} N$. So, we have $h_{N}=0$ if and only if $\nabla_{J N}^{\top} N=\kappa J N$ with $\kappa=h(J N, J N)$, i.e., $J N$ is a principal direction with characteristic curvature $\kappa$.

Lemma 5.1 Let $\Sigma \subset \mathbf{C} P^{n}$ be an oriented hypersurface with normal $N$, characteristic curvature $\kappa$, and $H=\operatorname{tr}(h)$. Then we have

$$
h_{i j} \Lambda_{\sigma_{j, n}}\left(X_{i}\right)=(H-\kappa) N+J h_{N} .
$$

Proof The proof is a computation based on the following relation between the second fundamental form $\sigma$ and the Weingarten endomorphism, that is a consequence of (3.19):

$$
\Lambda_{\sigma(X, Y)}(V)=2\langle X, Y\rangle V+\langle Y, V\rangle X+\langle X, V\rangle Y+\langle J Y, V\rangle J X+\langle J X, V\rangle J Y,
$$

for every $X, Y, V \in T_{A} \mathbf{C} P^{n}$. For details see [7, Section 1] and recall the normalization $g(A, B)=4\langle A, B\rangle$.

Indeed, by (5.2) we get:

$$
\begin{aligned}
\Lambda_{\sigma\left(X_{j}, N\right)}\left(X_{i}\right) & =\delta_{i j} N-\delta_{i n} J X_{j}, \quad j=1, \ldots, n ; \\
\Lambda_{\sigma\left(J X_{j}, N\right)}\left(X_{i}\right) & =-\delta_{i j} J N+\delta_{i n} X_{j}, \quad j=1, \ldots, n-1,
\end{aligned}
$$

for any $i=1, \ldots, n$, and

$$
\begin{aligned}
\Lambda_{\sigma\left(X_{j}, N\right)}\left(J X_{i}\right) & =\delta_{i j} J N, \quad j=1, \ldots, n ; \\
\Lambda_{\sigma\left(J X_{j}, N\right)}\left(J X_{i}\right) & =\delta_{i j} N, \quad j=1, \ldots, n-1,
\end{aligned}
$$

for every $i=1, \ldots, n-1$. So, adding up appropriately we prove the thesis.

The following lemma is a technical computation.

Lemma 5.2 Let $\Sigma \subset \mathbf{C} P^{n}$ be an oriented hypersurface with normal $N$. Letting, for any orthonormal frame of $T \Sigma$,

$$
S_{1}=\sigma\left(\sigma_{i, N}, \sigma_{i, N}\right)-4 \pi\left(\sigma_{i, N}, N\right) X_{i} \text { and } S_{2}=-\pi(N, N) \Delta A,
$$

we have the identity

$$
S_{1}+S_{2}=2 \operatorname{tr}(h) N-\operatorname{tr}(\sigma)+2(n-1) \sigma_{N} .
$$

The proof is postponed to the end of the section. In the previous section, we introduced the linear operator $\sigma h: T_{A} \Sigma \rightarrow \operatorname{End}\left(T_{A} \Sigma, T_{A}^{\perp} \mathbf{C} P^{n}\right)$. In the same way, we define the linear operator $\sigma h^{2}(X)[Y]=\sigma\left(h^{2}(X), Y\right)$, for $X, Y \in T_{A} \Sigma$. Its trace is

$$
\operatorname{tr}\left(\sigma h^{2}\right)=\sigma h^{2}\left(X_{j}\right)\left[X_{j}\right]=\sigma\left(h_{i j} h\left(X_{i}\right), X_{j}\right)=h_{i j} h_{i k} \sigma_{j k} \in T_{A}^{\perp} \mathbf{C} P^{n} .
$$

Now we are ready to prove the main formula of this section.

Theorem 5.3 Let $\Sigma \subset \mathbf{C} P^{n}$ be an orientable hypersurface with constant mean curvature and normal $N$. Then we have

$$
\Delta \sigma_{N}=4 \kappa N+2 \operatorname{tr}\left(\sigma h^{2}-\sigma\right)-2|h|^{2} \sigma_{N}-4 J h_{N},
$$

where $\kappa$ is the characteristic curvature of $\Sigma$. 
Proof We check formula (5.4) using normal coordinates at the point $A_{0} \in \Sigma$. Using (4.3), (3.17) and the short notation $\pi_{N}=\pi(N, N)$, we have

$$
\begin{aligned}
\Delta \sigma_{N}= & \nabla_{X_{i}} \nabla_{X_{i}} \sigma_{N}=\nabla_{X_{i}}\left(2 \pi\left(\nabla_{X_{i}} N, N\right)(I-2 A)-2 \pi_{N} X_{i}\right) \\
= & 2\left\{\sigma\left(\nabla_{X_{i}} \nabla_{X_{i}} N, N\right)+\sigma\left(\nabla_{X_{i}} N, \nabla_{X_{i}} N\right)-4 \pi\left(\nabla_{X_{i}} N, N\right) X_{i}-\pi_{N} \nabla_{X_{i}} X_{i}\right\} \\
= & 2\left\{\sigma(\Delta N, N)+2 \sigma\left(h_{i j} X_{j}+\sigma_{i, N}, h_{i k} X_{k}+\sigma_{i, N}\right)\right. \\
& \left.-4 \pi\left(h_{i j} X_{j}+\sigma_{i, N}, N\right) X_{i}-\pi_{N}\left(-h_{i i} N+\sigma_{i i}\right)\right\} \\
= & -2\left(|h|^{2}+2(n-1)\right) \sigma_{N}+2 h_{i j} h_{i k} \sigma_{j k}+ \\
& +4 h_{i j}\left\{\left(\sigma\left(\sigma_{i j}, N\right)+\sigma\left(X_{j}, \sigma_{i, N}\right)\right)-2 \pi_{j, N} X_{i}\right\} \\
& +2\left(\sigma\left(\sigma_{i, N}, \sigma_{i, N}\right)-4 \pi\left(\sigma_{i, N}, N\right) X_{i}\right)-2 \pi_{N} \Delta A .
\end{aligned}
$$

We used the identity $\sigma\left(\sigma_{N}, N\right)=0$. By (3.21) and (5.3), we have

$$
\begin{aligned}
\Delta \sigma_{N}= & -2\left(|h|^{2}+2(n-1)\right) \sigma_{N}+2 h_{i j} h_{i k} \sigma_{j k}-4 h_{i j} \Lambda_{\sigma_{j, N}}\left(X_{i}\right) \\
& +4(n-1) \sigma_{N}-2 \operatorname{tr}(\sigma)+4 \operatorname{tr}(h) N \\
= & 4 \operatorname{tr}(h) N-2 \operatorname{tr}(\sigma)-2|h|^{2} \sigma_{N}+2 h_{i j} h_{i k} \sigma_{j k}-4 h_{i j} \Lambda_{\sigma_{j, N}}\left(X_{i}\right) .
\end{aligned}
$$

By (5.1), this ends the proof.

Proof of Lemma 5.2 We check the formula at the point $A_{0} \in \Sigma$. Using formulas (3.8), (3.9), and (3.7), we obtain

$$
\begin{aligned}
S_{1} & =2 \pi_{i, N}(I-2 A) \pi_{i, N}(I-2 A)^{2}-4 \pi\left(\sigma_{i, N}, N\right) X_{i} \\
& =2\left[\pi_{i, N}^{2}(I-2 A)-2\left(\pi_{i, N}(I-2 A) N+N \pi_{i, N}(I-2 A)\right) X_{i}\right] \\
& =2\left\{\left[\pi_{i, N}^{2}-2\left(\pi_{i, N} N-N \pi_{i, N}\right) X_{i}\right](I-2 A)\right\} .
\end{aligned}
$$

A simple computation gives

$$
\begin{aligned}
& \pi_{i, N}^{2}\left(I-2 A_{0}\right)=-\delta_{i n}\left(E_{i n}+E_{n i}\right)+E_{n n}+E_{i i}, \\
& \pi_{\widehat{j}, N}^{2}\left(I-2 A_{0}\right)=E_{n n}+E_{j j},
\end{aligned}
$$

and also

$$
\begin{aligned}
& \left(\pi_{i, N} N X_{i}-N \pi_{i, N} X_{i}\right)\left(I-2 A_{0}\right)=-\delta_{i n} E_{n i}+E_{i i}+\left(1-\delta_{i n}\right) A_{0}, \\
& \left(\pi_{\hat{j}, N} N \widehat{X}_{j}-N \pi_{\hat{j}, N} \widehat{X}_{j}\right)\left(I-2 A_{0}\right)=E_{j j}+A_{0} .
\end{aligned}
$$

Therefore, at $A_{0}$ we have

$$
\begin{aligned}
S_{1}= & 2 \sum_{i=1}^{n}\left[-\delta_{i n}\left(E_{i n}+E_{n i}\right)+E_{n n}+E_{i i}-2\left(-\delta_{i n} E_{n i}+E_{i i}+\left(1-\delta_{i n}\right) A_{0}\right)\right]+ \\
& +2 \sum_{i=1}^{n-1}\left[E_{n n}+E_{i i}-2\left(E_{i i}+A_{0}\right)\right] \\
= & 2\left[2 n E_{n n}-2\left(I-A_{0}\right)+(4-4 n) A_{0}\right] \\
= & 4 n E_{n n}-4 I+(12-8 n) A_{0} .
\end{aligned}
$$

Moreover, using (3.18) and $\sigma_{N}=2\left(E_{n n}-A_{0}\right)$, we have $4 I=\operatorname{tr}(\sigma)+4(n+1) A_{0}$ and $2 E_{n n}=\sigma_{N}+2 A_{0}$, and hence, we get

$$
S_{1}=2 n \sigma_{N}-\operatorname{tr}(\sigma)+8(1-n) A_{0} .
$$


Now, we compute $S_{2}$ at $A_{0}$. Using $\pi_{N}=2 N, \pi_{N} \operatorname{tr}(\sigma)=-8 n A_{0}+8 E_{n n}$, and $\pi_{N} \sigma_{N}=$ $-4 A_{0}+4 E_{n n}$, we get

$$
\begin{aligned}
S_{2} & =-\pi_{N}\left(-\operatorname{tr}(h) N+\operatorname{tr}(\sigma)-\sigma_{N}\right) \\
& =-\left(-2 \operatorname{tr}(h) N-8 n A_{0}+8 E_{n n}+4 A_{0}-4 E_{n n}\right) \\
& =2 \operatorname{tr}(h) N-8(1-n) A_{0}-2 \sigma_{N} .
\end{aligned}
$$

Adding $S_{1}$ and $S_{2}$, we get the claim.

\section{Trace of the second variation of the area}

Let $\Sigma$ be a $C^{\infty}$ hypersurface with normal $N$ and without boundary, and let $u \in C^{\infty}(\Sigma)$ be a function with zero mean:

$$
\int_{\Sigma} u \mathrm{~d} \mu=0
$$

where $\mu$ is the Riemannian hypersurface measure in $\mathbf{C} P^{n}$. For $t \in \mathbb{R}$ and $p \in \Sigma$, let $t \mapsto \gamma_{p}(t)$ be the curve (geodesic) in $\mathbf{C} P^{n}$ solving $\nabla_{\dot{\gamma}}^{\top} \dot{\gamma}=0$ with $\gamma(0)=p$ and $\dot{\gamma}(0)=u(p) N(p)$. For small $t$, the hypersurface $\Sigma(t ; u)=\left\{\gamma_{p}(t) \in \mathbf{C} P^{n}: p \in \Sigma\right\}$ is well defined and we denote its area by $\mathscr{A}(t ; u)=\mu(\Sigma(t ; u))$. If we have

$$
\left.\frac{\mathrm{d} \mathscr{A}(t ; u)}{\mathrm{d} t}\right|_{t=0}=0
$$

for any $u \in C^{\infty}(\Sigma)$ satisfying (6.1), then $\Sigma$ has constant mean curvature. If $\Sigma(t ; u)$ is the boundary of a region with volume $V(t)$, then condition (6.1) implies that $V^{\prime}(0)=0$.

The second variation of the area functional is given by the formula

$$
\mathscr{A}^{\prime \prime}(u)=\left.\frac{\mathrm{d}^{2} \mathscr{A}(t ; u)}{\mathrm{d} t^{2}}\right|_{t=0}=-\int_{\Sigma} u \mathscr{L} u \mathrm{~d} \mu,
$$

where

$$
\mathscr{L} u=\Delta u+\left(|h|^{2}+\operatorname{Ric}(N)\right) u
$$

is the Jacobi operator, see e.g. [2]. It is well known that the Ricci curvature on unit vectors is a geometric constant in $\mathbf{C} P^{n}$, and namely $\operatorname{Ric}(N)=2 n+2$.

Definition 6.1 (Stability) We say that an oriented hypersurfaces $\Sigma$ without boundary and with constant mean curvature is stable if $\mathscr{A}^{\prime \prime}(u) \geq 0$ for any $u \in C^{\infty}(\Sigma)$ satisfying (6.1).

On $\Sigma$ we consider the matrix valued function $u=\Delta A$, where $A$ is the position matrix. For any $V \in H^{n+1}$, we define the scalarization $u_{V}=\langle u, V\rangle$. By the divergence theorem, the function $u_{V}$ satisfies the zero-mean condition (6.1) because $\Sigma$ has no boundary.

The mapping $Q_{\Sigma}: H^{n+1} \rightarrow \mathbb{R}$ defined by $Q_{\Sigma}(V)=\mathscr{A}^{\prime \prime}\left(u_{V}\right)$ is a quadratic form. If the surface $\Sigma$ is stable then $Q_{\Sigma}$ is positive semidefinite, i.e., $Q_{\Sigma}(V) \geq 0$ for any $V \in H^{n+1}$. It follows that $\operatorname{tr}\left(Q_{\Sigma}\right) \geq 0$. In the next theorem we compute an explicit expression for this trace.

Theorem 6.2 Let $\Sigma$ be an oriented complete hypersurface with constant mean curvature. The trace of the quadratic form $Q_{\Sigma}$ is

$$
\operatorname{tr}\left(Q_{\Sigma}\right)=4 \int_{\Sigma}\left\{2(n+1) H^{2}+2\left(n^{2}-1\right)\left(2 n-|h|^{2}\right)-(H+\kappa)^{2}-\left|h_{N}\right|^{2}\right\} \mathrm{d} \mu .
$$


Proof For any orthonormal basis $\mathscr{V}$ of $H^{n+1}$, we have

$$
\begin{aligned}
\operatorname{tr}\left(Q_{\Sigma}\right) & =\sum_{V \in \mathscr{V}} Q_{\Sigma}(V)=-\int_{\Sigma} \sum_{V \in \mathscr{V}} u_{V} \mathscr{L} u_{V} \mathrm{~d} \mu \\
& =-\int_{\Sigma}\left\{\langle u, \Delta u\rangle+\left(|h|^{2}+2(n+1)\right)|u|^{2}\right\} \mathrm{d} \mu .
\end{aligned}
$$

We compute first the norm of $u$. By formula (4.1) we have:

$$
\begin{aligned}
|u|^{2}=|\Delta A|^{2} & =\left\langle\mathscr{H}-\sigma_{N}-\operatorname{tr}(h) N, \mathscr{H}-\sigma_{N}-\operatorname{tr}(h) N\right\rangle \\
& =H^{2}+|\mathscr{H}|^{2}-2\left\langle\mathscr{H}, \sigma_{N}\right\rangle+\left|\sigma_{N}\right|^{2},
\end{aligned}
$$

because the matrices $N$ and $\mathscr{H}-\sigma_{N}$ are orthogonal. Using the identity $\sigma(X, Y)=$ $\sigma(J X, J Y)$ for any $X, Y \in T_{A} \mathbf{C} P^{n}$ (see [6, Proposition 1.6]), we obtain

$$
|\mathscr{H}|^{2}=\sum_{i, j=1}^{2 n}\left\langle\sigma_{i i}, \sigma_{j j}\right\rangle=4 \sum_{i, j=1}^{n}\left\langle\sigma_{i i}, \sigma_{j j}\right\rangle .
$$

Now by (3.19) we have for any $i, j=1, \ldots, n$

$$
\left\langle\sigma_{i i}, \sigma_{j j}\right\rangle=2\left(1+\delta_{i j}\right)
$$

and hence,

$$
\begin{aligned}
|\mathscr{H}|^{2} & =4 \sum_{i, j=1}^{n} 2\left(1+\delta_{i j}\right)=8\left(n^{2}+\sum_{i, j=1}^{n} \delta_{i j}\right) \\
& =8\left(n^{2}+n\right)=8 n(n+1) .
\end{aligned}
$$

In the same way, we have

$$
\left\langle\mathscr{H}, \sigma_{N}\right\rangle=\sum_{i=1}^{2 n}\left\langle\sigma_{i i}, \sigma_{N}\right\rangle=2 \sum_{i=1}^{n}\left\langle\sigma_{i i}, \sigma_{N}\right\rangle=4 \sum_{i=1}^{n}\left(1+\delta_{i n}\right)=4(n+1),
$$

where we used (6.4) with $j=n$. Finally, by (6.4) with $i=j=n$, we have

$$
\left|\sigma_{N}\right|^{2}=4
$$

Now, by (6.5), (6.6) and (6.7) we get

$$
\begin{aligned}
|u|^{2} & =H^{2}+8 n(n+1)-8(n+1)+4=H^{2}+8(n+1)(n-1)+4 \\
& =H^{2}+8 n^{2}-8+4=H^{2}+4\left(2 n^{2}-1\right) .
\end{aligned}
$$

By formula (3.18), we have $\Delta \mathscr{H}=-4(n+1) \Delta A=-4(n+1) u$. Hence, from formula (4.1) we find

$$
\begin{aligned}
\langle u, \Delta u\rangle & =\left\langle u,-H \Delta N-4(n+1) u-\Delta \sigma_{N}\right\rangle \\
& =-4(n+1)|u|^{2}-H\langle u, \Delta N\rangle-\left\langle u, \Delta \sigma_{N}\right\rangle .
\end{aligned}
$$

Since $N$ is orthogonal to $\mathscr{H}, \sigma_{N}$, and $\sigma_{i j}$, we have

$$
\begin{aligned}
\langle u, \Delta N\rangle= & \left\langle-\operatorname{tr}(h) N+\mathscr{H}-\sigma_{N},-\left(|h|^{2}+2(n-1)\right) N+2 h_{i j} \sigma_{i j}-\operatorname{tr}(h) \sigma_{N}\right\rangle \\
= & H\left(|h|^{2}+2(n-1)\right)+2\langle\mathscr{H}, \operatorname{tr}(\sigma h)\rangle-H\left\langle\mathscr{H}, \sigma_{N}\right\rangle \\
& -2\left\langle\sigma_{N}, \operatorname{tr}(\sigma h)\right\rangle+H\left|\sigma_{N}\right|^{2} .
\end{aligned}
$$


By (3.19) we have

$$
\begin{aligned}
& \left\langle\sigma\left(X_{i}, X_{i}\right), \sigma\left(X_{j}, X_{k}\right)\right\rangle=2\left(\delta_{j k}+\delta_{i k} \delta_{i j}\right), \\
& \left\langle\sigma\left(X_{i}, X_{i}\right), \sigma\left(X_{j}, J X_{k}\right)\right\rangle=0,
\end{aligned}
$$

for every $i, j, k=1, \ldots, n$. Hence, by (6.9) and (6.10) and also using the notation $h_{\hat{\jmath} \hat{k}}:=$ $h\left(J X_{j}, J X_{k}\right)$, we get

$$
\begin{aligned}
\langle\mathscr{H}, \operatorname{tr}(\sigma h)\rangle & =2 \sum_{i=1}^{n} \sum_{j, k=1}^{2 n-1} h_{j k}\left\langle\sigma_{i i}, \sigma_{j k}\right\rangle= \\
& =4 \sum_{i, j, k=1}^{n} h_{j k}\left(\delta_{j k}+\delta_{i k} \delta_{i j}\right)+4 \sum_{i=1}^{n} \sum_{j, k=1}^{n-1} h_{\hat{\jmath} \hat{k}}\left(\delta_{j k}+\delta_{i k} \delta_{i j}\right) \\
& =4 n h_{j j}+4 h_{i i}=4(n+1) H .
\end{aligned}
$$

Again by (6.9) and (6.10),

$$
\left\langle\sigma_{N}, \operatorname{tr}(\sigma h)\right\rangle=h_{i j}\left\langle\sigma_{N}, \sigma_{i j}\right\rangle=2 h_{i j}\left(\delta_{i j}+\delta_{j n} \delta_{i n}\right)=2 H+2 \kappa .
$$

Finally, using (6.6), (6.7), (6.11) and (6.12), we get

$$
\begin{aligned}
\langle u, \Delta N\rangle & =H\left(|h|^{2}+2(n-1)\right)+8(n+1) H-4(n+1) H-4(H+\kappa)+4 H \\
& =\left(|h|^{2}+6 n+2\right) H-4 \kappa,
\end{aligned}
$$

and so we obtain the formula

$$
\langle u, \Delta N\rangle=H\left(|h|^{2}+6 n+2\right)-4 \kappa .
$$

We are left with the computation of $\left\langle u, \Delta \sigma_{N}\right\rangle$. By formula (5.4), also using $\left\langle u, h_{N}\right\rangle=0$, we obtain

$$
\begin{aligned}
\left\langle u, \Delta \sigma_{N}\right\rangle= & \left\langle-\operatorname{tr}(h) N+\mathscr{H}-\sigma_{N}, 4 \kappa N-2 \mathscr{H}-2|h|^{2} \sigma_{N}+2 \operatorname{tr}\left(\sigma h^{2}\right)\right\rangle \\
= & -4 \kappa H-2|\mathscr{H}|^{2}-2\left(|h|^{2}-1\right)\left\langle\mathscr{H}, \sigma_{N}\right\rangle+2\left\langle\mathscr{H}, \operatorname{tr}\left(\sigma h^{2}\right)\right\rangle+2|h|^{2}\left|\sigma_{N}\right|^{2} \\
& -2\left\langle\sigma_{N}, \operatorname{tr}\left(\sigma h^{2}\right)\right\rangle .
\end{aligned}
$$

Again by (6.9) and (6.10),

$$
\begin{aligned}
\left\langle\mathscr{H}, \operatorname{tr}\left(\sigma h^{2}\right)\right\rangle & =2 \sum_{i=1}^{n} \sum_{\ell, j, k=1}^{2 n-1} h_{\ell j} h_{\ell k}\left\langle\sigma_{i i}, \sigma_{j k}\right\rangle= \\
& =4 \sum_{i=1}^{n} \sum_{\ell=1}^{2 n-1}\left\{\sum_{j, k=1}^{n} h_{\ell j} h_{\ell k}\left(\delta_{j k}+\delta_{i k} \delta_{i j}\right)+\sum_{j, k=1}^{n-1} h_{\ell j} h_{\ell \hat{k}}\left(\delta_{j k}+\delta_{i k} \delta_{i j}\right)\right\} \\
& =4 n \sum_{\ell, j=1}^{2 n-1} h_{\ell j}^{2}+4 \sum_{\ell, i=1}^{2 n-1} h_{\ell i}^{2}=4(n+1)|h|^{2} .
\end{aligned}
$$

So, we get

$$
\left\langle\mathscr{H}, \operatorname{tr}\left(\sigma h^{2}\right)\right\rangle=4(n+1)|h|^{2} .
$$


Moreover, we have

$$
\begin{aligned}
\left\langle\sigma_{N}, \operatorname{tr}\left(\sigma h^{2}\right)\right\rangle & =h_{i j} h_{i k}\left\langle\sigma_{N}, \sigma_{j k}\right\rangle=2 h_{i j} h_{i k}\left(\delta_{j k}+\delta_{k n} \delta_{j n}\right) \\
& =2 h_{i j}^{2}+2 h_{i n}^{2}=2|h|^{2}+2\left(\left|h_{N}\right|^{2}+\kappa^{2}\right) .
\end{aligned}
$$

Adding (6.5), (6.6), (6.7), (6.14) and (6.15), we get the identity

$$
\left\langle u, \Delta \sigma_{N}\right\rangle=4|h|^{2}-8(2 n-1)(n+1)-4 H \kappa-4 \kappa^{2}-4\left|h_{N}\right|^{2} .
$$

Now, in order to get (6.3), we just have to use formulas (6.8), (6.13) and (6.16) and sum them up.

In the next lemmas, we test the trace formula (6.3) on geodesic spheres and on the tubes introduced in Sect. 2.

Lemma 6.3 For the sphere $\Sigma_{r} \subset \mathbf{C} P^{n}$, we have $\operatorname{tr}\left(Q_{\Sigma_{r}}\right) \geq 0$ if and only if $\tan ^{2} r \leq 2 n+1$. The trace is zero if and only if $\tan ^{2} r=2 n+1$.

Proof Letting $t=\tan r$, by formulas (2.1), we have:

$$
\begin{aligned}
& |h|^{2}=(2 n-1) \frac{1}{t^{2}}+t^{2}-2, \\
& H^{2}=(2 n-1)^{2} \frac{1}{t^{2}}+t^{2}-2(2 n-1), \\
& (H+\kappa)^{2}=4(n-1)^{2} \frac{1}{t^{2}}+4 t^{2}-8(n-1) .
\end{aligned}
$$

Inserting these values into the trace formula (6.3), we find

$$
\operatorname{tr}\left(Q_{\Sigma_{r}}\right)=-\frac{8 n(n-1)}{t^{2}} \mu\left(\Sigma_{r}\right)\left\{t^{4}-2 n t^{2}-(2 n+1)\right\} .
$$

Then we have $\operatorname{tr}\left(Q_{\Sigma_{r}}\right) \geq 0$ if and only if $t^{4}-2 n t^{2}-(2 n+1) \leq 0$, which holds if and only if $t^{2} \leq 2 n+1$. In particular, the trace is zero precisely when $t^{2}=2 n+1$.

Lemma 6.3 shows that formula (6.3) detects the sharp stability interval for the radius of a geodesic sphere, see Theorem 2.2. In the next lemma, we consider the tubes $T_{r}^{k}$.

Lemma 6.4 Let $k=1, \ldots, n-1$. For the tubes $T_{r}^{k} \subset \mathbf{C} P^{n}$, we have:

(1) If $n=2 k+1$, then $\operatorname{tr}\left(Q_{T_{r}^{k}}\right)>0$ for any $0<r<\pi / 2$.

(2) If $n \neq 2 k+1$, then $\operatorname{tr}\left(Q_{T_{r}^{k}}\right) \geq 0$ if and only if $\tan ^{2} r \leq d(n, k)$ for a certain positive number $d(n, k)$ that satisfies

$$
d(n, k)>\frac{2 n-2 k+1}{2 k+1} .
$$

Moreover, $\operatorname{tr}\left(Q_{T_{r}^{k}}\right)=0$ precisely when $\tan ^{2} r=d(n, k)$.

Proof We use the short notation $t=\tan r$ and $m=2 k+1$. By formulas (2.2), we have:

$$
\begin{aligned}
& |h|^{2}=m t^{2}+(2 n-m) \frac{1}{t^{2}}-2, \\
& H^{2}=m^{2} t^{2}+(2 n-m)^{2} \frac{1}{t^{2}}-2 m(2 n-m), \\
& (H+\kappa)^{2}=(m+1)^{2} t^{2}+(2 n-m+1)^{2} \frac{1}{t^{2}}-2(m+1)(2 n-m+1) .
\end{aligned}
$$


Inserting these values into formula (6.3), we get the following expression for the trace of $Q_{T_{r}^{k}}$

$$
\operatorname{tr}\left(Q_{T_{r}^{k}}\right)=\frac{8 \mu\left(T_{r}^{k}\right)}{t^{2}}\left(a t^{4}+b t^{2}+c\right)
$$

where $a, b, c$ are coefficients depending on $n$ and $k$, and namely

$$
\begin{aligned}
a & =m(n+1)(m-n+1)-\frac{1}{2}(m+1)^{2}, \\
b / 2 & =(n+1)\left[\left(n^{2}-1\right)-m(2 n-m)\right]+\frac{1}{2}(m+1)(2 n-m+1), \\
c & =(n+1)(2 n-m)(n-m+1)-\frac{1}{2}(2 n-m+1)^{2} .
\end{aligned}
$$

It is easy to check that $b=a+c$, which means that $t^{2}=-1$ is a root of $a t^{4}+b t^{2}+c=0$. So, we have the decomposition

$$
\operatorname{tr}\left(Q_{T_{r}^{k}}\right)=\frac{8 \mu\left(T_{r}^{k}\right)}{t^{2}}\left(t^{2}+1\right)\left(a t^{2}+c\right)
$$

Now there are two cases: $n=m$ and $n \neq m$. When $n=m=2 k+1$, then $n$ must be odd and in this case it is $a=c=\frac{1}{2}\left(n^{2}-1\right)>0$. It follows that $\operatorname{tr}\left(Q_{T_{r}^{k}}\right)>0$ for any $t>0$.

In the case $n \neq m$, we have $a<0$ and $c>0$. We conclude that $\operatorname{tr}\left(Q_{T_{r}^{k}}\right) \geq 0$ if and only if $t^{2} \leq d(n, k):=-c / a$. After some computations, inequality (6.17) is equivalent to $m>1$, that is $k>0$.

Lemma 6.4 shows that Theorem 6.3 is not sharp in the case of the tubes $T_{r}^{k}$. In fact, when $\tan ^{2} r=d(n, k)$, then the trace of $Q_{T_{r}^{k}}$ is zero, but the tube $T_{r}^{k}$ is unstable by Theorem 2.2 part 2.

We finish this section proving the non-stability of the tubes $V_{r}$ around $\mathbf{R} P^{n}$.

Lemma 6.5 The tube $V_{r} \subset \mathbf{C} P^{n}$ is unstable for any $r>0$.

Proof Using formulas (2.3), we compute:

$$
\begin{aligned}
|h|^{2} & =(n-1)\left(t^{2}+\frac{1}{t^{2}}\right)+\frac{16 t^{2}}{\left(1-t^{2}\right)^{2}}, \\
H^{2} & =(n-1)^{2} \frac{\left(1-t^{2}\right)^{2}}{t^{2}}+\frac{16 t^{2}}{\left(1-t^{2}\right)^{2}}-8(n-1), \\
(H+\kappa)^{2} & =(n-1)^{2} \frac{\left(1-t^{2}\right)^{2}}{t^{2}}+\frac{64 t^{2}}{\left(1-t^{2}\right)^{2}}-16(n-1) .
\end{aligned}
$$

Inserting these values into formula (6.3), we obtain the value for the trace of $Q_{V_{r}}$

$$
\operatorname{tr}\left(Q_{V_{r}}\right)=-4(n-1) \mu\left(V_{r}\right)\left\{(n-1)\left(t^{2}+\frac{1}{t^{2}}\right)+\frac{32 n t^{2}}{\left(1-t^{2}\right)^{2}}+2(5 n-1)\right\},
$$

and we see that $\operatorname{tr}\left(Q_{V_{r}}\right)<0$ for any $t=\tan r \in(0,1)$ and $n \geq 2$. We deduce that the surfaces $V_{r}$ are not stable. 


\section{Proof of Theorems 1.1 and 1.2}

In this section, we prove Theorems 1.1 and 1.2. Let $\Sigma \subset \mathbf{C} P^{n}$ be a complete stable oriented hypersurface that is connected.

Proof of Theorem 1.1 We denote by $\widehat{h}$ the restriction of the second fundamental form $h$ of $\Sigma$ to the complex tangent space $\mathbf{C} T \Sigma$ and by $\widehat{H}$ the trace of $\widehat{h}$. At any point of $\Sigma$, we have the identities

$$
H=\widehat{H}+\kappa \text { and }|h|^{2}=|\widehat{h}|^{2}+2\left|h_{N}\right|^{2}+\kappa^{2},
$$

and the inequalities

$$
|h|^{2} \geq|\widehat{h}|^{2}+\kappa^{2} \text { and } \mid \widehat{h}^{2} \geq \frac{\widehat{H}^{2}}{2(n-1)}=\frac{(H-\kappa)^{2}}{2(n-1)} .
$$

Inserting these inequalities and $\left|h_{N}\right| \geq 0$ into (6.3), we obtain

$$
\begin{aligned}
\operatorname{tr}\left(Q_{\Sigma}\right) & \leq 4 \int_{\Sigma}\left\{2(n+1) H^{2}+2\left(n^{2}-1\right)\left(2 n-\kappa^{2}-\frac{(H-\kappa)^{2}}{2(n-1)}\right)-(H+\kappa)^{2}\right\} \mathrm{d} \mu \\
& =-4 n \int_{\Sigma} p(\kappa ; H, n) \mathrm{d} \mu,
\end{aligned}
$$

where $p(\cdot ; H, n)$ is the polynomial in (1.1). By our assumption $p(\kappa ; H, n) \geq 0$ on $\Sigma$, we deduce that $\operatorname{tr}\left(Q_{\Sigma}\right) \leq 0$. On the other hand, the stability of $\Sigma$ implies that $\operatorname{tr}\left(Q_{\Sigma}\right) \geq 0$. We deduce that $\operatorname{tr}\left(Q_{\Sigma}\right)=0$ and that we have equality in (7.2). In turn, the equality in (7.2) implies that $p(\kappa ; H, n)=0$, that

$$
|h|^{2}=|\widehat{h}|^{2}+\kappa^{2} \quad \text { and } \quad|\widehat{h}|^{2}=\frac{\widehat{H}^{2}}{2(n-1)},
$$

and also that $h_{N}=0$ on $\Sigma$.

The equation $h_{N}=0$ means that $J N$ is an eigenvector of $h$. By Maeda's theorem [4], this implies that the characteristic curvature $\kappa$ is constant. This also simply follows from the fact that $\kappa$ is one of the roots of $p(\kappa ; H, n)=0$. Here we use the fact that $\Sigma$ is connected.

The identity in the right-hand side of (7.3) implies that $\Sigma$ is umbilical in $\mathbf{C} T \Sigma$, i.e., each unit vector in $\mathbf{C} T \Sigma$ is an eigenvector of $h$ with eigenvalue $\lambda=\widehat{H} / 2(n-1)$. Moreover, $\lambda$ is constant on $\Sigma$, because $\widehat{H}=H-\kappa$ is constant.

The two constants $\kappa$ and $\lambda$ are different, because in $\mathbf{C} P^{n}$ there are no totally umbilical hypersurfaces. By Takagi's theorem, Proposition 2.1, $\Sigma$ is a geodesic sphere: up to a suitable choice of the center of the sphere, we have $\Sigma=\Sigma_{r}$ for some $r \in(0, \pi / 2)$. By Lemma 6.3 the equation $\operatorname{tr}\left(Q_{\Sigma_{r}}\right)=0$ implies that $\tan ^{2} r=2 n+1$.

Finally, we prove Theorem 1.2. We shall use Takagi's characterization of tubes in Proposition 2.3 and the computations of Lemmas 6.4 and 6.5.

Proof of Theorem 1.2 We have the decomposition $\mathbf{C} T \Sigma=T_{1} \oplus T_{2}$ where, at each point of $\Sigma, T_{1}$ and $T_{2}$ are subspaces of real dimension $\alpha$ and $\beta=2(n-1)-\alpha$. We denote by $h_{1}$ and $h_{2}$ the restrictions of $h$ to $T_{1}$ and $T_{2}$, respectively, and we let $H_{1}=\operatorname{tr}\left(h_{1}\right)$ and $H_{2}=\operatorname{tr}\left(h_{2}\right)$.

We have the identity $H=H_{1}+H_{2}+\kappa$ and the inequalities

$$
|h|^{2} \geq\left|h_{1}\right|^{2}+\left|h_{2}\right|^{2}+\kappa^{2}, \quad\left|h_{1}\right|^{2} \geq \frac{H_{1}^{2}}{\alpha} \quad \text { and } \quad\left|h_{2}\right|^{2} \geq \frac{H_{2}^{2}}{\beta} .
$$


Inserting these inequalities and $\left|h_{N}\right| \geq 0$ into (6.3), we obtain

$$
\operatorname{tr}\left(Q_{\Sigma}\right) \leq-8\left(n^{2}-1\right) \int_{\Sigma} p\left(H_{1}, H_{2} ; H, n, \alpha\right) \mathrm{d} \mu,
$$

where $p(\cdot ; H, n, \alpha)$ is the polynomial in (1.3). By our assumption $p\left(H_{1}, H_{2} ; H, n, \alpha\right) \geq 0$ on $\Sigma$, we deduce that $\operatorname{tr}\left(Q_{\Sigma}\right) \leq 0$. On the other hand, the stability of $\Sigma$ implies that $\operatorname{tr}\left(Q_{\Sigma}\right) \geq 0$. We deduce that $\operatorname{tr}\left(Q_{\Sigma}\right)=0$ and that we have equality in (7.5). In turn, the equality in (7.5) implies that $p\left(H_{1}, H_{2} ; H, n, \alpha\right)=0$, that

$$
|h|^{2}=\left|h_{1}\right|^{2}+\left|h_{2}\right|^{2}+\kappa^{2} \quad \text { and } \quad\left|h_{1}\right|^{2}=\frac{H_{1}^{2}}{\alpha}, \quad\left|h_{2}\right|^{2}=\frac{H_{2}^{2}}{\beta}
$$

and also that $h_{N}=0$ on $\Sigma$.

The equation $h_{N}=0$ means that $J N$ is an eigenvector of $h$. By Maeda's theorem [4], the characteristic curvature $\kappa$ is constant.

The identities in (7.6) imply that $T_{1}$ is an eigenspace of $h$ for a curvature $\lambda_{1}$, and $T_{2}$ is an eigenspace of $h$ for a curvature $\lambda_{2}$. We clearly have $H_{1}=\alpha \lambda_{1}$ and $H_{2}=\beta \lambda_{2}$. From $p\left(H_{1}, H_{2} ; H, n, \alpha\right)=0$ and $H_{1}+H_{2}=$ constant, we deduce that $\lambda_{1}$ and $\lambda_{2}$ are constant.

Now we have three cases:

(1) $\kappa=\lambda_{1}=\lambda_{2}$. This case is empty, because in $\mathbf{C} P^{n}$ there are no totally umbilical hypersurfaces.

(2) Precisely two of the numbers $\kappa, \lambda_{1}$, and $\lambda_{2}$ are equal. By Proposition $2.1, \Sigma$ is a geodesic sphere. Hence, it must be $\lambda_{1}=\lambda_{2}$ and the radius of the sphere is $\tan ^{2} r=2 n+1$, as explained at the end of the proof of Theorem 1.1.

(3) The three numbers $\kappa, \lambda_{1}$, and $\lambda_{2}$ are different. By Proposition 2.3, the surface $\Sigma$ is either a tube around $\mathbf{C} P^{k}, \Sigma=T_{r}^{k}$ with $\alpha=2 k$ even, or a tube around $\mathbf{R} P^{n}, \Sigma=V_{r}$ with $\alpha=n-1$. The latter case $\Sigma=V_{r}$ is excluded because $V_{r}$ is unstable for any $r>0$, by Proposition 6.5. We are left with the case $\Sigma=T_{r}^{k}$ with $\alpha=2 k$ and for some $r>0$. The radius is determined by the equation $\operatorname{tr}\left(Q_{T_{r}^{k}}\right)=0$. However, this equation either has no solution (this happens in the case $\alpha=\beta$ ), or its unique solution $r>0$ has the property that the tube $T_{k}^{r}$ is unstable, as shown in Proposition 6.4.

The only possible case is that $\Sigma$ is a geodesic sphere with radius $\tan ^{2} r=2 n+1$.

\section{References}

1. Barbosa, J.L., do Carmo, M.: Stability of hypersurfaces with constant mean curvature. Math. Z. 185(3), 339-353 (1984)

2. Barbosa, J.L., do Carmo, M., Eschenburg, J.: Stability of hypersurfaces of constant mean curvature in Riemannian manifolds. Math. Z. 197(1), 123-138 (1988)

3. Cecil, T.E., Ryan, P.J.: Focal sets and real hypersurfaces in complex projective space. Trans. Am. Math. Soc. 269(2), 481-499 (1982)

4. Maeda, Y.: On real hypersurfaces of a complex projective space. J. Math. Soc. Jpn. 28(3), 529-540 (1976)

5. Nomizu, K.: Some results in E. Cartan's theory of isoparametric families of hypersurfaces. Bull. Am. Math. Soc. 79, 1184-1188 (1973)

6. Ros, A.: Spectral geometry of CR-minimal submanifolds in the complex projective space. Kodai Math. J. 6(1), 88-99 (1983)

7. Ros, A.: On spectral geometry of Kähler submanifolds. J. Math. Soc. Jpn. 36(3), 433-448 (1984)

8. Tai, S.-S.: Minimum imbeddings of compact symmetric spaces of rank one. J. Differ. Geom. 2, 55-66 (1968)

9. Takagi, R.: Real hypersurfaces in a complex projective space with constant principal curvatures. J. Math. Soc. Jpn. 27, 43-53 (1975) 
10. Takagi, R.: Real hypersurfaces in a complex projective space with constant principal curvatures. II. J. Math. Soc. Jpn. 27(4), 507-516 (1975)

Publisher's Note Springer Nature remains neutral with regard to jurisdictional claims in published maps and institutional affiliations. 